\title{
Algunas nOtas SOBRE PUTNAM Y LA MECÁNICA CUÁNTICA ${ }^{1}$
}

\author{
SOME NOTES ON PUTNAM AND QUANTUM MECHANICS
}

Javier García Sanz

\begin{abstract}
RESUMEN
Este artículo revisa las aportaciones más relevantes de Hilary Putnam a la interpretación de la mecánica cuántica, en especial al llamado "problema de la medida". Discutimos sus ideas sobre la aparente no aplicabilidad de las reglas cuánticas al mundo macroscópico y la aparición de la regla de Born, que determina la naturaleza probabilista del mundo cuántico. Algunos de los estudios sobre lógica de Putnam son asimismo revisados debido a su especial relevancia en el nuevo campo de la computación cuántica.
\end{abstract}

PALABRAS CLAVE: Putnam, mecánica cuántica, problema de la medida, computación cuántica.

\begin{abstract}
This article reviews the most relevant contributions made by Hilary Putnam to the interpretation of Quantum Mechanics, specifically to the so-called "measurement problem". We discuss his ideas regarding the apparent non-applicability of the quantum rules to the macroscopic world and the emergence of the Born rule, which determines the probabilistic nature of the quantum world. Some of the logical studies of Putnam are also reviewed due to their special relevance to the novel field of quantum computation.
\end{abstract}

KEYWORDS: Putnam, Quantum mechanics, measurement problem, quantum computation.

1 Nota del editor: Tras el fallecimiento del autor de este artículo, el editor de esta sección monográfica de Análisis ha contado, en la preparación del texto para su publicación, con la amable colaboración de Javier Rodríguez Laguna, quien también ha aportado el abstract. 
Como no podía ser menos en un discípulo de Reichenbach y Carnap, Hilary Putnam mostró gran interés tanto por la interpretación como por la estructura lógica de la mecánica cuántica. Desde sus primeros trabajos hubo grandes cambios en estos temas y él tuvo oportunidad de revisar sus ideas sobre los mismos. Hoy día estos temas son objeto de abundantes discusiones. El propósito de estas notas no es tanto resumirlas sino ver si desarrollos teóricos más recientes apoyan o refutan algunas ideas fundamentales planteadas.

Contaba Putnam que en 1962 mantuvo varias conversaciones con un famoso físico teórico. ${ }^{2}$ Este físico le decía que los filósofos habían inventado un problema inexistente con la interpretación de la mecánica cuántica. No había tal problema, insistía, porque Bohr lo había dejado todo claro con la llamada interpretación de Copenhague. Años más tarde el mismo físico le reconoció que sí que había un problema. "Si pudiese dedicarle unos meses lo dejaría resuelto". Más aún, en un congreso internacional afirmó que lejos de dejarlo claro, Bohr había lavado el cerebro a toda una generación de físicos (Putnam 2005).

El problema "inexistente" era lo que algunos llamaban el problema de la medida. En mecánica cuántica el estado de un sistema está representado por una función de onda normalizada que puede considerarse como un vector de módulo unidad en un espacio de Hilbert complejo. A su vez, cada magnitud física (un observable) está representada por un operador lineal autoadjunto cuyos vectores propios constituyen una base del espacio de Hilbert. Normalmente el estado cuántico de un sistema no es ninguno de estos vectores propios sino una combinación lineal de los mismos que evoluciona de forma continua de acuerdo con la ecuación de Schrödinger. Solo cuando se hace una "medida" de un observable se interrumpe la evolución continua y el sistema "colapsa" a uno de los vectores propios del operador que representa a la magnitud medida. Pero el proceso de medida era aleatorio: nadie sabía cómo actuaba, en qué instante preciso tenía lugar o cómo "sabía" el sistema qué magnitud se estaba midiendo y en qué base tenía que colapsar.

Para Putnam la propia expresión "problema de la medida" era inaceptable, no solo en mecánica cuántica sino en cualquier teoría científica. Las medidas eran una subclase de interacciones físicas, pero las medidas no podían obedecer leyes diferentes de las que obedecen todas las interacciones físicas y en consecuencia "medida" nunca podía ser un término indefinido en una teoría física satisfactoria. Por ello Putnam prefería la expresión problema del colapso, que no tenía que ser desencadenado necesariamente por una interacción con un sistema físico mayor.

2 Aunque Putnam no lo cita explícitamente, no hay duda de que este físico era Murray Gell-Mann. 
El postulado fundamental de la mecánica cuántica, que establecía la relación entre el formalismo matemático y los resultados experimentales, era la regla de Born, que daba la probabilidad de que el sistema quedara en un estado dado tras el colapso. Cualquier observable cuántico, representado por un operador lineal autoadjunto $\hat{A}$ tenía un conjunto de estados propios $\phi_{n}$ y valores propios correspondientes $\lambda_{n}$ tales que $\hat{A} \phi_{n}=\lambda_{n} \phi_{n}$. Dichos estados propios constituían una base de un espacio de Hilbert, de modo que la función de onda $\psi$ del sistema podía representarse siempre por una combinación lineal de estados propios $\phi_{n}$ con coeficientes $c_{n}$ complejos

$$
|\Psi\rangle=\sum_{n=1}^{D} c_{n}\left|\phi_{n}\right\rangle \quad \text { con } \sum_{n=1}^{D}\left|c_{n}\right|^{2}=1
$$

La regla de Born afirmaba que la probabilidad de que el sistema físico quedara en un estado $\phi_{i}$ tras la medida era precisamente el cuadrado del valor absoluto del producto escalar del estado $\psi$ del sistema y el estado $\phi_{i}$; es decir, el cuadrado del valor absoluto del coeficiente que multiplicaba al estado $\phi_{i}$ en la combinación.

$$
P\left(\lambda_{i}\right)=\left|\left(\Psi \mid \phi_{i}\right)\right|^{2}=\left|c_{i}\right|^{2}
$$

Aunque tales valores eran probabilidades, no estaba claro cuál era la relación con la teoría general de la probabilidad ni había una deducción de la regla a partir de principios más fundamentales.

En 1957 Andrew Gleason demostró que de acuerdo con la estructura del espacio de Hilbert, y para espacios de dimensión igual o mayor que 3, la forma más general de asignar probabilidades a los estados cuánticos era a través de la traza de un operador autoadjunto (Gleason 1957). La regla de Born sería así una simple consecuencia del teorema de Gleason.

En 1968 James Hartle intentó demostrar la regla de Born desde una interpretación frecuencial de la probabilidad. Según Hartle "el resultado de una medida de la magnitud $A$ no puede predecirse para ningún sistema individual. La magnitud $A$ no está en general perfectamente definida en el estado $\psi$. (....) Pero ahora podemos demostrar que las frecuencias de los resultados pueden predecirse para conjuntos infinitos de sistemas preparados de forma idéntica. De esta forma las predicciones estadísticas de la mecánica cuántica se deducirán de afirmaciones no estadísticas sobre sistemas individuales" (Hartle 1968). Tales sistemas idénticamente preparados podían ser los muchos universos de Hugh Everett. Según la interpretación de Everett cada vez que se hacía una medida el universo se di- 
vidía en tantos universos como resultados posibles tuviera la medida, es decir, la dimensión $D$ del espacio de Hilbert correspondiente: la función de onda no colapsaba sino que se transformaba en una suma de funciones cada una de las cuales describía uno de estos universos separados (Everett 1957). Según Hartle, al hacer una segunda medida de la misma magnitud cada uno de estos universos volvía a dividirse en otros $D$ universos. Al cabo de $N$ medidas sucesivas habría $D^{N}$ universos o historias posibles, cada una de las cuales estaba definida por una serie de $N$ resultados. La probabilidad de un resultado sería entonces el número de historias en las que aparecía tal resultado dividido por el número total de historias. En términos más precisos el valor que daba la regla de Born era el valor propio de un operador frecuencia que actuaba sobre un espacio de Hilbert que era un producto tensorial de $N$ espacios de Hilbert (Hartle 1968). Argumentos similares con ligeras variantes fueron propuestos por Farhi, Goldstone y Gutmann (Farhi et al. 1989). Sin embargo estos argumentos han estado sometidos a fuertes críticas. En particular, no hay una extensión unívoca a $N$ infinito de un espacio producto tensorial de $N$ espacios (Caves y Schack 2005).

Putnam tampoco creía que la regla de Born pudiera justificarse de esta forma. El cálculo de la frecuencia era un simple problema de combinatoria para el que solo se necesitaba el teorema del binomio. De hecho, él pensaba que en la mayoría de las historias aparecería cualquier resultado posible de la medida y afirmaba que en la interpretación de los muchos universos la mecánica cuántica sería la primera teoría física que predecía que las observaciones de la mayoría de los observadores estarían en desacuerdo con la teoría (Putnam 2005).

Otro problema importante en la interpretación de la teoría cuántica era explicar por qué el comportamiento cuántico estaba confinado al mundo submicroscópico y no se manifestaba en el mundo macroscópico (algo que ya había sido enfatizado por Schrödinger con su famoso ejemplo del gato que debería quedar en un estado superpuesto, mitad vivo y mitad muerto). El propio Bohr había reconocido que los aparatos de medida debían obedecer a la física clásica. También Putnam era consciente de este problema y pedía una teoría cuántica que distinguiera entre micro-observables y macro-observables. "El resultado que queremos es que aunque los micro-observables no tienen necesariamente valores numéricos definidos en todo instante, los macro-observables sí los tienen. Y queremos que este resultado salga de la mecánica cuántica de forma natural. No queremos añadirlo simplemente a la mecánica cuántica como un principio ad hoc. Sin embargo, los intentos de deducir este resultado han sido hasta ahora completamente infructuosos (...). 'Macro-observable' no es el tipo de término que pueda ser un primiti- 
vo irreducible en una teoría física final, de modo que yo invoqué alguna extensión futura de la mecánica cuántica que explicaría por qué los macro-observables nunca pueden encontrarse en estados superpuestos tales como $(1 / \sqrt{ } 2) \mid$ gato vivo $\rangle+$ $(1 / \sqrt{ } 2) \mid$ gato muerto)" (Putnam 2005). "Difícilmente podemos remitirnos a una teoría (la física clásica) en la axiomatización de otra si se supone que la primera teoría es incorrecta y la segunda está diseñada para reemplazarla" (Putnam 1975). ${ }^{3}$

Y añadía, "Una alternativa más razonable podría ser la siguiente: definamos una medida como una interacción en la que un Sistema A (el Sistema 'medido'), que previamente estaba aislado, interactúa con un Sistema B (el Sistema 'que mide') de tal manera que produce un cambio en B que afecta a algún 'macro-observable' —alguna propiedad macroscópicamente observable de B. Esta definición es, por supuesto, incompleta. Necesitamos también que se nos diga cómo interpretar las medidas - i-e. cómo determinar qué significa el cambio en una propiedad concreta macroscópicamente observable de B. Es posible hacerlo, sin embargo, y de una manera que no se salga del formalismo de la propia mecánica cuántica. Así podríamos llegar a una formalización de la mecánica cuántica (incluida la interpretación estadística mínima) que no contenga el término 'medida' como primitivo. Pero el término 'macro-observable' aparecerá ahora como primitivo. Quizá, sin embargo, este término se mostrará más fácil de definir. Parece plausible que los macro-observables puedan definirse como ciertos promedios de grandes números de micro-observables en sistemas muy grandes (...) La cuestión a la que nos enfrentamos es si a partir de tal caracterización mecano-cuántica de un macroobservable, junto con las leyes de la mecánica cuántica, es posible deducir que los macro-observables retienen siempre valores precisos haya o no una interacción de medida que los implique. Si podemos hacerlo, entonces la apariencia de una paradoja y el carácter ad hoc de la interpretación de Copenhague desaparecerán".

En definitiva, Bohr, Schrödinger, Landau y Putnam, entre otros, requerían la existencia de un dominio macroscópico donde los objetos estuvieran en estados

${ }^{3}$ Esta misma paradoja aparece perfectamente expresada en el capítulo primero de la Mecánica Cuántica de Landau:

De ordinario, la teoría más general se puede formular de manera lógicamente cerrada con independencia de una teoría menos general que constituye un caso límite de la primera. (...) La formulación de las tesis fundamentales de la mecánica cuántica, en cambio, es en principio imposible sin acudir a la mecánica clásica.

La mecánica cuántica ocupa así una posición muy particular en el conjunto de las teorías físicas: contiene la mecánica clásica como caso límite y al mismo tiempo tiene necesidad de este caso límite para su propia fundamentación. 
perfectamente definidos y dejara de ser válido el principio de superposición. Sin embargo, en 1985 A. Leggett y A. Garg mostraron que las predicciones de la mecánica cuántica eran incompatibles con dos hipótesis que denominaron "realismo macroscópico" y "medibilidad no invasiva en el nivel macroscópico" (Leggett y Garg 1985). Leggett y Garg dedujeron un conjunto de desigualdades (posteriormente conocidas como desigualdades de Bell en el tiempo) que acotan ciertas combinaciones lineales de medidas de correlación en dos instantes. Desde entonces varios experimentos mostraron violaciones de estas desigualdades que indicaban la existencia de estados superpuestos en objetos macroscópicos. Las mayores violaciones se observaron en un experimento realizado en 2015. El experimento consistía en provocar un camino aleatorio de un átomo de cesio en un potencial periódico. Las correlaciones entre las posiciones del átomo en instantes diferentes eran inexplicables si el átomo siguiera trayectorias clásicas, y exigían trayectorias "cuánticas" a través de estados superpuestos (Robens et al. 2015).

$$
* * *
$$

Para terminar estas notas me gustaría añadir algún comentario no tanto sobre las interpretaciones de la mecánica cuántica por parte de Putnam sino sobre sus aportaciones a algunos problemas lógicos susceptibles de ser tratados con algoritmos cuánticos. Como es sabido, en los años 20 del siglo pasado, David Hilbert, en su disputa con el intuicionismo de Brower, propuso que cualquier teoría matemática debería ir acompañada de un procedimiento de decisión efectivo, es decir, un algoritmo que permitiera decidir en un número finito de pasos la verdad o falsedad de cualquier expresión correctamente formulada dentro de la teoría. Nació así el denominado problema de la decisión o Enstcheidungproblem. También es sabido que en 1931 Gödel puso fin al sueño de Hilbert al demostrar que había proposiciones indecidibles. Lo mismo hicieron Church y Turing unos años más tarde. ${ }^{4}$ El problema se reducía ahora a establecer si los algoritmos de decisión efectivos podían ejecutarse en tiempo polinómico, es decir, si el número de pasos necesario para llegar a la solución dependía polinómicamente de la longitud o el número de variables de la expresión a decidir, o si eran de tiempo no polinómico (básicamente exponencial). Así, los problemas son de tipo polinómico, $P$, si puede encontrarse una solución en tiempo polinómico y NP si un candidato a solución

${ }^{4}$ El propio Putnam contribuyó años más tarde de forma decisiva a demostrar que el décimo problema de Hilbert, la existencia de solución para una ecuación diofántica general, era indecidible. 
puede comprobarse en tiempo polinómico. Nació así el problema conocido como $P \neq N P$ que pretende establecer si realmente existen problemas NP para los que nunca podría encontrarse un algoritmo en tiempo polinómico o si simplemente el desconocimiento de dichos algoritmos era debido a que no habíamos sido capaces de encontrarlos hasta ahora. Una variante de este problema consistía en averiguar si un problema NP podría resolverse en tiempo polinómico cuando se implementaba en una máquina que obedece a leyes cuánticas. En esta línea parece apuntar el algoritmo cuántico para la descomposición de un número en factores primos diseñado por Peter Shor (Shor 1997).

Otro problema NP típico era el problema de la satisfactibilidad booleana, es decir, saber si dada una expresión booleana con variables y sin cuantificadores existe una asignación de valores para sus variables que haga que la expresión sea verdadera. Obviamente la longitud de una simple tabla de verdad para una expresión dependería exponencialmente del número de variables incluidas en la misma. En 1971 Stephen Cook demostró que el problema de la satisfactibilidad era un problema NP completo. En 1960 Martin Davis y Hilary Putnam habían introducido un algoritmo clásico para tratar el problema de la satisfactibilidad booleana. El algoritmo consistía en expresar la fórmula booleana en forma normal conjuntiva y considerar iterativamente las variables y eliminarlas mediante la resolución de cada cláusula en la que la variable es afirmada con una cláusula en la que es negada.

Más recientemente este problema ha sido estudiado como un ejemplo susceptible de ser tratado mediante computación cuántica adiabática (Farhi et al. 2000). Una expresión lógica en forma normal conjuntiva puede traducirse en un hamiltoniano cuántico que es una suma de hamiltonianos sencillos cada uno de ellos correspondiente a una de las cláusulas de la forma. La computación cuántica adiabática consiste en partir de un hamiltoniano cuyo estado fundamental es conocido e ir modificando lentamente el hamiltoniano hasta llegar a la expresión deseada. Si la modificación es suficientemente lenta el teorema adiabático asegura que el sistema seguirá en todo momento en su estado fundamental y así la obtención del estado fundamental del hamiltoniano final nos permite conocer la verdad o falsedad de la expresión. Una forma equivalente de tratar el problema es mediante el método conocido como cristalización cuántica (quantum annealing) que busca el mínimo absoluto de una función de varias variables expresada como una suma de funciones enteras no negativas cada una de las cuales depende solamente de unas pocas variables. Algunos experimentos numéricos permiten estimar que estos algoritmos cuánticos reducen notablemente el tiempo de ejecución comparados con algoritmos clásicos aplicados a los mismos problemas, aunque siempre dentro de 
tiempos polinómicos (Shor 1997). Se ha sugerido no obstante que en algunos casos la reducción podría ser mucho más notable (Farhi et al. 2000). Aunque no parece muy probable que esto resuelva definitivamente el problema $P \neq N P$, el algoritmo puede ser fundamental para la demostración de teoremas en lógica matemática.

$$
\text { Javier García Sanz. }
$$

UNED

\section{BIBLIOGRAFÍA}

CAVES, C. y R. SCHACK (2005): "Properties of the frequency operator do not imply the quantum probability postulate", Ann. Phys.

Davis, M. y H. PUTNAM (1970): "A Computing Procedure for Quantification Theory", Journal of the $A C M, 7,201-215$.

Everett III, H. (1957) “'Relative State' Formulation of Quantum Mechanics”, Rev. Mod. Pbys., 29, 454.

FARHI, E., J. GOLDSTONE, y S. GUTMANN (1989): "How probability arises in quantum mechanics", Ann. Phys., 192, 368.

FARHi, E., J. Goldstone, S. GutMann, M. Sipser (2000): “Quantum Computation by Adiabatic Evolution", arXiv:quantum-ph/0001106.

Gleason, A. M. (1957): "Measures on the Closed Subspaces of a Hilbert Space”, J. Math. Mech., 6, 885.

Hartle, J. B. (1968): “Quantum Mechanics of Individual Systems”, Am. J. Phys., 36, 704.

LegGeTt, A. J., y A. Garg (1985): "Quantum Mechanics versus Macroscopic Realism: Is the Flux There when Nobody Looks?”, Phys. Rev. Lett., 54.

Putnam, H. (1975): "A Philosopher Looks at Quantum Mechanics", en H. Putnam, Philosophical Papers, vol. 1, Mathematics, Matter and Method, Cambridge: Cambridge University Press, pp. 130-58.

Putnam, H. (2005): "A Philosopher Looks at Quantum Mechanics (Again)", Brit. J. Phil. Sci., 56 (2005), 615-634.

Robens, C., W. Alt, D. Meschede, C. Emary y A. Alberti (2015): "Ideal Negative Measurements in Quantum Walks Disprove Theories Based on Classical Trajectories", Physical Review X, 5, 011003.

SHOR, P. (1997): "Polynomial-Time Algorithms for Prime Factorization and Discrete Logarithms on a Quantum Computer", SLAM J. Sci. Statist. Comput. 26, 1484. 